\title{
An NIRS-based assay of chemical composition and biomass digestibility for rapid selection of Jerusalem artichoke clones
}

Meng Li1,2, Siyang He ${ }^{1,2}$, Jun Wang ${ }^{1,2}$, Zuxin Liü ${ }^{3}$ and Guang Hui Xie ${ }^{1,2^{*}}$

\begin{abstract}
Background: High-throughput evaluation of lignocellulosic biomass feedstock quality is the key to the successful commercialization of bioethanol production. Currently, wet chemical methods for the determination of chemical composition and biomass digestibility are expensive and time-consuming, thus hindering comprehensive feedstock quality assessments based on these biomass specifications. To find the ideal bioethanol feedstock, we perform a nearinfrared spectroscopic (NIRS) assay to rapidly and comprehensively analyze the chemical composition and biomass digestibility of 59 Jerusalem artichoke (Helianthus tuberosus L., abbreviated JA) clones collected from 24 provinces in six regions of China.

Results: The distinct geographical distribution of JA accessions generated varied chemical composition as well as related biomass digestibility (after soluble sugars extraction and mild alkali pretreatment). Notably, the soluble sugars, cellulose, hemicellulose, lignin, ash, and released hexoses, pentoses, and total carbohydrates were rapidly and perfectly predicted by partial least squares regression coupled with model population analyses (MPA), which exhibited significantly higher predictive performance than controls. Subsequently, grey relational grade analysis was employed to correlate chemical composition and biomass digestibility with feedstock quality score (FQS), resulting in the assignment of tested JA clones to five feedstock quality grades (FQGs). Ultimately, the FQGs of JA clones were successfully classified using partial least squares-discriminant analysis model coupled with MPA, attaining a significantly higher correct rate of $97.8 \%$ in the calibration subset and $91.1 \%$ in the validation subset.
\end{abstract}

Conclusions: Based on the diversity of JA clones, the present study has not only rapidly and precisely examined the biomass composition and digestibility with MPA-optimized NIRS models but has also selected the ideal JA clones according to FQS. This method provides a new insight into the selection of ideal bioethanol feedstock for high-efficiency bioethanol production.

Keywords: Jerusalem artichoke, Chemical composition, Chemical pretreatment, Biomass digestibility, Near-infrared spectroscopy, Grey relational grade analysis

\section{Background}

In recent years, fossil fuels consumption and greenhouse gas emissions have increased dramatically in step with rapid global industrialization, especially in China. Compared with developed economies, energy consumption in China has increased considerably in 2016 [1],

\footnotetext{
*Correspondence: xiegh@cau.edu.cn

${ }^{1}$ College of Agronomy and Biotechnology, China Agricultural University, Beijing 100193, China

Full list of author information is available at the end of the article
}

accounting for $23 \%$ of global energy consumption and $27 \%$ of increased global energy demand [2]. In addition, estimated carbon emissions in China have grown by more than $75 \%$ since 2004 [2]. Consequently, the combination of China's huge base energy consumption rate and its dramatically increasing energy demand is forcing its government to address energy needs and find sources of cleaner energy [1]. Bioenergy derived from lignocellulosic biomass currently holds great promise for addressing these energy and environmental concerns, due to large 
biomass reserves, resource reproducibility, low resource replacement costs, and low bioenergy production impact on carbon balance [3]. Moreover, lignocellulosic biomass is transformable into various forms of energy, such as briquettes, biogas, and bioethanol, making it an ideal substitute for fossil fuels [4-6].

Jerusalem artichoke (Helianthus tuberosus L., abbreviated JA), a perennial crop related to the sunflower, is widely distributed and cultivated in China and exhibits cold resistance, drought resistance, and salt tolerance [6-9]. Recently, numerous studies have demonstrated JA to be one of the most promising bioenergy crops for bioethanol production [6]. Indeed, JA tuber contains a considerable amount of inulin that is easily fermented into biofuel [10], while JA stem contains an abundance of lignocellulose that is available post-harvest $[9,11]$. Paradoxically, despite a long history of JA tuber use for biofuel production, JA stem has been largely overlooked for this application and investigation of its use as feedstock for bioethanol production is warranted $[6,11,12]$. Principal chemical components of JA stem include soluble sugars, cellulose, hemicellulose, lignin, and ash, which vary widely among cultivated varieties [13]. Notably, variability in biomass major chemical constituents and physical structural attributes can lead to biomass recalcitrance when these attributes act in concert to negatively influence lignocellulosic biomass usability [14, 15]. Therefore, both principal chemical composition and biomass digestibility as feedstock quality specifications should be considered together for selecting ideal JA accessions. In addition, the ideal bioethanol feedstock could be identified with high levels of soluble sugars, cellulose, and hemicellulose, low levels of lignin and ash, and high biomass digestibility [13-15]. Grey relational grade analysis (GRA), which employs matrix calculation to quantify data at different relational levels and transforms soluble sugars, cellulose, hemicellulose, lignin, and ash contents and biomass digestibility into a single grey relational grade [16], could permit JA stem feedstock quality to be comprehensively and fairly evaluated by assigning a unique feedstock quality score (FQS). In order to meet the demands of industry, the FQS values of JA were further assigned to different feedstock quality grades (FQG).

High-throughput methodologies are often necessary for screening large numbers of lignocellulosic biomass samples [17]. Recently, NIRS coupled with multivariate calibrations such as partial least squares regression (PLSR) and partial least squares-discriminant analysis (PLS-DA) has rapidly become a key method for addressing this problem [17-21]. As the most common analytical approach, PLS has been widely demonstrated in quantitative analysis to obtain accurate and reliable results comparable to wet chemical methods [17, 22].
On the other hand, since the difference in physical and chemical properties between different raw materials has great influence on the conversion performance of biofuels, it is important to distinguish different plant varieties [23]. In this sector, several attempts have been made to address this problem [24]. So far, however, there has been little discussion about the application of NIRS in JA biomass for quantitative prediction of chemical composition and biomass digestibility and qualitative analysis of germplasm resources [17, 23, 25]. While it is universally accepted that PLS cannot completely solve data overfitting of NIRS data, a serious hindrance to the application and promotion of this technology, variable selection techniques have subsequently emerged as powerful platforms for addressing this issue [26]. Meanwhile, a recent advance in molecular spectroscopy known as model population analysis (MPA) has been demonstrated to overcome this problem $[27,28]$. As the most famous MPA algorithm, variable selection using competitive adaptive reweighted sampling (CARS), allows for selection of an optimal variable subset existing within the full spectra that can be coupled to PLSR to generate a model based on the simple, but effective "survival of the fittest" principle of Darwin's Evolution Theory [29]. In addition, the random frog (RF) method, originally proposed for gene selection and disease classification, has recently become an efficient reversible jump Markov Chain Monte Carlolike approach that further enhances variable selection [30]. Therefore, it is essential to evaluate the MPA-optimized PLSR or MPA-optimized PLS-DA models for use in high-throughput qualitative and quantitative analysis of JA stem biomass.

In this study, we conducted a series analysis of 59 representative JA clones collected from six regions of China for chemical composition, biomass digestibility, and NIRS results. Based on the reliable physical and chemical data, the MPA-optimized PLSR models were developed for rapidly and precisely predicting the chemical composition and biomass digestibility of JA clones. To obtain ideal bioethanol feedstocks, GRA model was performed to comprehensively evaluate JA stem samples by FQS so that the nationwide JA clones could be fairly assessed and assigned to different FQGs. Finally, the MPA-optimized PLS-DA models are developed for rapid and accurate classification of FQGs across the nationwide JA population.

\section{Results}

Variations of chemical composition and biomass digestibility of JA accessions for NIRS

In 2006, after JA was characterized as a potential bioenergy crop, the National Energy R\&D Center for Non-food Biomass initiated a long-term project for nationwide JA 
germplasm resources collection and identification [7-9, 11]. As a result, a total of 59 representative JA accessions were selected from 24 provinces with distinctive environments and climates (Fig. 1). According to the National Bureau of Statistics of China, these geographic areas could be divided into six regions including Northeast China (NEC), North China (NC), East China (EC), Central-South China (CSC), Northwest China (NWC), and Southwest China (SWC) [1]. Due to their distinct geographic locations and variable genotypes, these JA clones exhibited remarkable variations either in phenotype or in yield performance, demonstrating high potential for use in bioenergy crop genetic modification or high-quality biomass feedstock selection $[7-9,31]$. Therefore, these JA accessions could be deemed an ideal sample population for the analysis of cell wall chemical and physical characteristics.

Sample diversity was clearly demonstrated by the varying levels of soluble sugars, cellulose, hemicellulose, lignin, and ash in JA stem samples (Fig. 2a). On the one hand, it was obvious that JA stem contains a relatively high level of soluble sugars, cellulose, and hemicellulose, with mean values of $18.2 \%, 28.3 \%$, and $14.0 \%$, respectively. Among JA samples from all investigated regions, those from SWC displayed significantly lower cellulose content (22.5-29.5\%), while significant higher hemicellulose level was found in JA samples from NEC (13.1-18.4\%) $(P<0.001)$. On the other hand, both ash and lignin levels in JA stem were relatively lower than those in other bioenergy feedstocks [17], although JA samples from NEC contained a significantly higher lignin level than did JA samples from other regions $(P<0.001)$. Notably, the JA population studied here exhibited relatively higher levels of fermentable carbohydrates with lower levels of lignin and ash, indicating a relatively lower biomass recalcitrance than that observed for other feedstocks [15, 31]. In this study, biomass digestibility was defined by accounting for

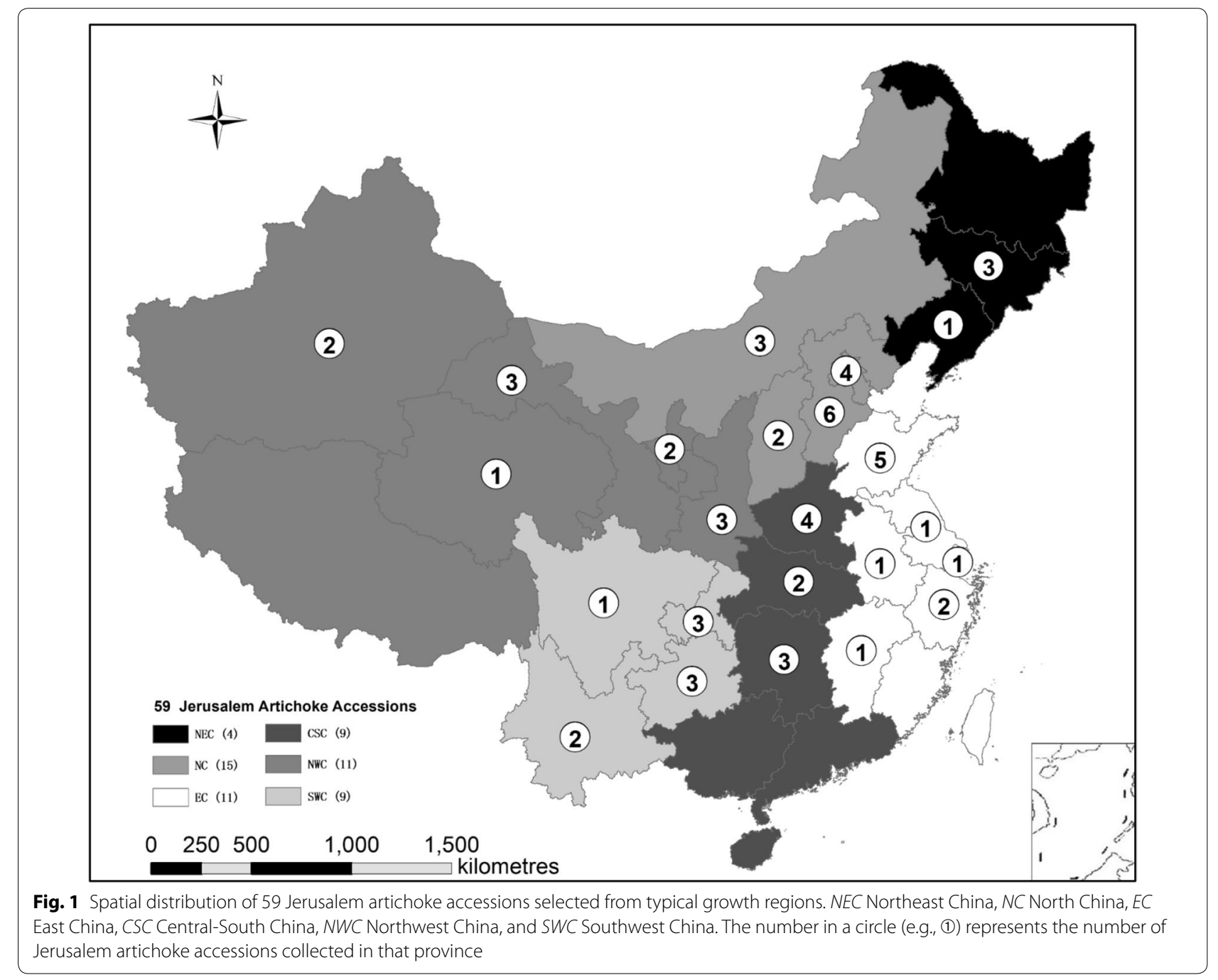




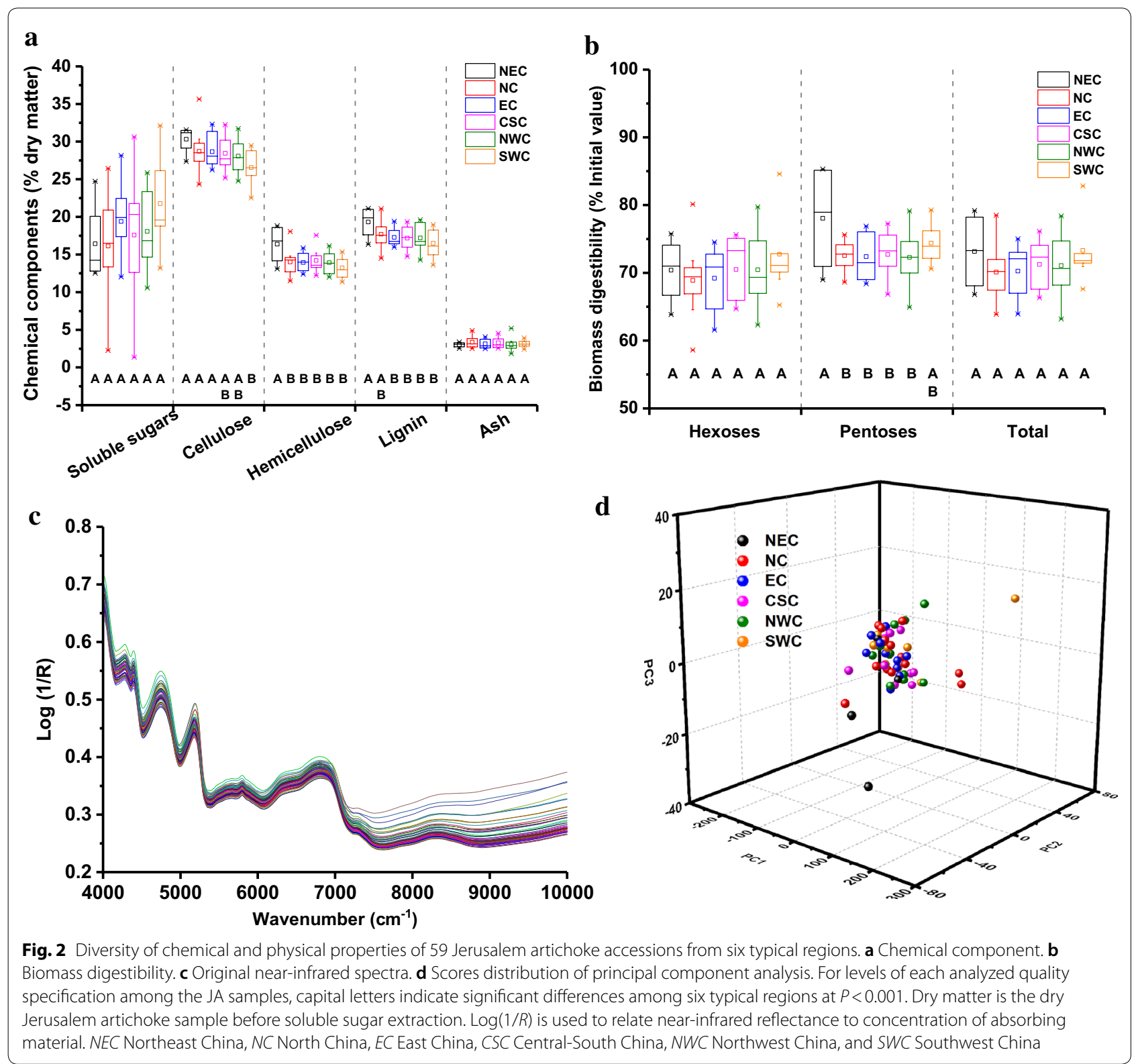

hexoses, pentoses, and total carbohydrates generated from soluble sugars-extracted JA samples after a combined alkali-based pretreatment and saccharification assay [32]. As expected, a large variation of biomass digestibility was observed among the sample population (Fig. 2b). Specifically, yields of hexoses ranged from 58.6 to $84.6 \%$ after pretreatment and subsequent enzymatic hydrolysis, while yields of pentoses ranged from 64.9 to $85.3 \%$, leading to yields of total carbohydrates ranging from 63.2 to $82.8 \%$ among assayed JA clones. Meanwhile, ANOVA analysis demonstrated that there were no significant differences in biomass digestibility among JA clones collected from various regions, except for the yield of pentose released from JA clones from NEC. Hence, this extensive collection of JA samples exhibits high levels of desired components and biomass digestibility, indicating that JA stem could be considered as an ideal feedstock for biofuel production.

NIRS, recorded from 10,000 to $4000 \mathrm{~cm}^{-1}$ with a resolution of $4 \mathrm{~cm}^{-1}$, displayed high absorption intensity with obvious baseline discrepancies and reflectance peak shifts (Fig. 2c). It is well known that the strong peaks observed in different spectral regions are attributable to frequency doubling and frequency combining characteristics of vibrations of hydrogen-containing molecules [24, 33]. Thus, the corresponding intensity of these peaks could 
be used to obtain information regarding biological characteristics, physical structure, and chemical composition of biomass [24, 25]. In general, the main absorption band peaks occurred within the range from 4000 to $7500 \mathrm{~cm}^{-1}$, demonstrating species-level similarity among JA clones. In this study, principal component analysis (PCA) was further developed for sample comparison, as well as to identify outliers. The PCA relies on projecting spectral variables on several reconstructed variables which are representative of original NIRS variation [34]. As shown in Fig. 2d, the NIRS plots of the 59 JA clones displayed a uniformly mixed and symmetrical distribution with respect to the three principal components (accounting for $93.3 \%$ of spectral variance), indicating that JA samples from six typical regions may have variable cell wall chemical and physical characteristics. Consequently, it was shown that the 59 sampled JA populations displayed relatively diverse cell wall chemical and physical characteristics, all of which would be suitable for NIRS modeling. To clearly explain the comprehensive assessment, a brief overview of is available in Fig. 3.

\section{Optimization of spectral variable selection and samples sets partitioning}

Judicious selection of spectral information is a crucial step for successful NIRS modeling, which not only permits the collection of strong informative variables but also removes interference due to uninformative variables
[23, 26]. In this study, two MPA algorithms including CARS and RF were employed for spectral variable selection of chemical components and biomass digestibility of JA clones. Obviously different spectral variable sets were generated using these two MPA algorithms, which closely reflected their very different theoretical underpinnings (Fig. 4). In general, the populations of spectral variable sets selected by CARS were generally larger than those selected by RF, except for hexoses and pentoses sets. It has been reported that the strong peak at approximately $5150-5195 \mathrm{~cm}^{-1}$, a peak primarily attributed to $\mathrm{O}-\mathrm{H}$ asymmetric stretching and $\mathrm{O}-\mathrm{H}$ deformation bands of water, was believed to interfere with the prediction of other bond species [17, 23]. Interestingly, both CARS and RF successfully avoided the selection of variables from this spectral region in this study. This result could be due to that most absorption bands of cell wall polymers were correlated with the vibrations from $\mathrm{O}-\mathrm{H}$ groups, which could be strongly interfered by the absorption of water and further lead to poorer correlationship between the discussed spectral region and cell wall polymers [22, 23, $34]$. For the prediction of soluble sugars, cellulose, and hemicellulose, the most important spectral regions were identified at 4015-4022, 4285-4296, 4392-4412, 47604780 , 5776-5796, 6329-6336, 6775-6822, and 7305$7328 \mathrm{~cm}^{-1}[24,34,35]$. Meanwhile, lignin was identified at $4015-4022,4392-4412$, and $5776-5796 \mathrm{~cm}^{-1}$ according to the stretching vibration $(\mathrm{O}-\mathrm{H}, \mathrm{C}-\mathrm{H}, \mathrm{C}-\mathrm{O}$, and

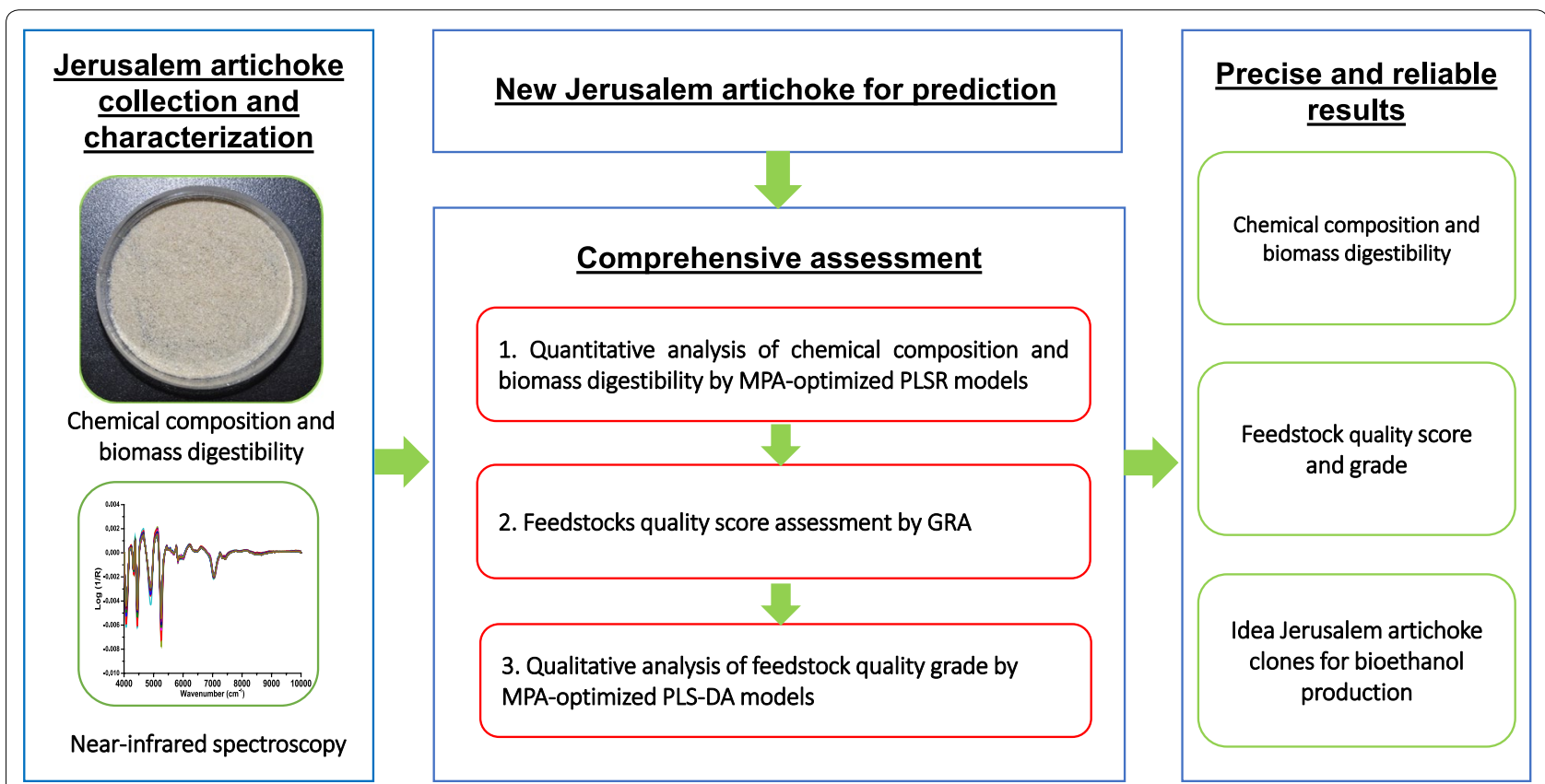

Fig. 3 Flowchart of the comprehensive assessment for ideal Jerusalem artichoke bioethanol feedstock. MPA model population analysis, PLSR partial least squares regression, PLS-DA partial least squares-discriminant analysis, GRA grey relational grade analysis 


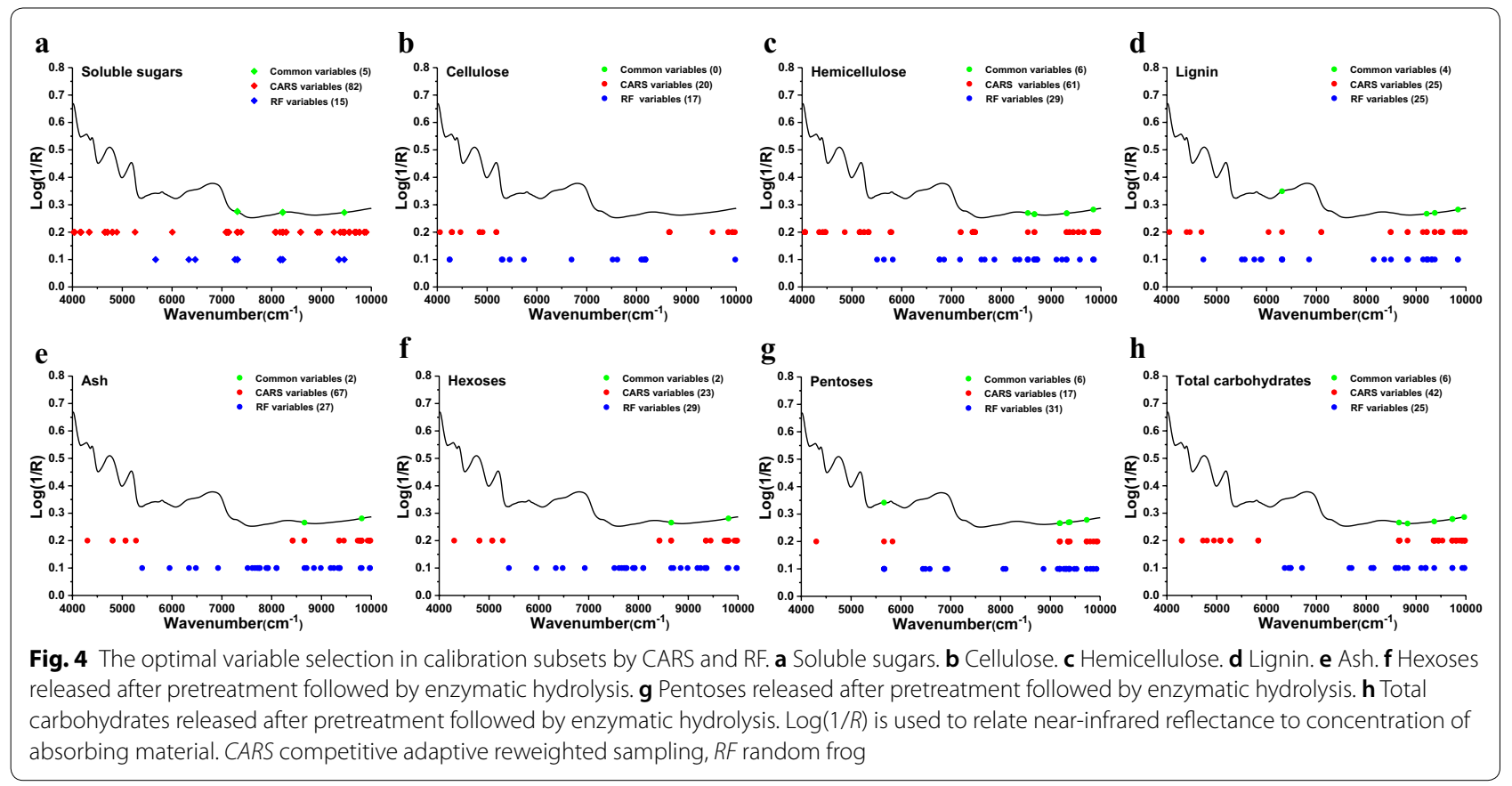

$\mathrm{C}-\mathrm{C})$ and the overtone stretching band of $\mathrm{O}-\mathrm{H}[22,23]$. Notably, the MPA algorithms that were employed exhibited significant efficiency in variable selection within the aforementioned spectral regions (Fig. $4 a-d$ ). As previously reported, ash and biomass digestibility could be indirectly predicted by identifying the types of adjacent organic bonds within the biomass sample. Nevertheless, very few NIRS studies have been used to identify specific spectral regions that were predictive for this characteristic $[17,23,25]$. In the present study, very different spectral variable sets were successfully obtained using these two MPA algorithms for prediction of ash and biomass digestibility (Fig. 4e-h). Although they made up only a small proportion of the full spectra consisting of 1557 variables, these selected variables could effectively reduce the high collinearity of NIRS to overcome the defect of PLS [29].

It is well known that strong multivariate calibration relies heavily on both representative calibration subsets and external validation subsets [23]. In this study, the Kennard-Stone (KS) algorithm was employed for sample subsets partitioning due to its past frequent use for this purpose. For accurate and robust multivariate calibration, one of every five samples was included in the validation subset based on full spectra and two kinds of characteristic spectra, while the remaining samples were used for the calibration subset. As shown in Additional file 1: Fig. S1, the solid lines and dashed lines superimposed upon each histogram represent normal distributions that were used to delineate the discrepancy between each histogram and normality. The broad range of values for each specification could largely be attributed to the ranges in JA native spatial geographical distribution and genotypes. In general, for calibration and validation of chemical components and biomass digestibility of 59 JA accessions, histograms based on full spectra, CARSoptimized spectra, and RF-optimized spectra displayed relatively broad and approximately normal distributions. Moreover, most calibration subsets showed nearly the same distributions as the corresponding validation subsets, whereas all lacked distinct bimodal, skewed, or uniform distributions. Meanwhile, similar results were found in principal component plots distributions based on full spectra, CARS-optimized spectra, and RF-optimized spectra (Additional file 1: Fig. S2) and the 3D score plots of NIRS data from both calibration and validation subsets were well mixed and displayed relatively symmetrical distributions. Therefore, KS algorithms facilitated the optimization of both the calibration subset and related validation subset, making them suitable for subsequent multivariate calibrations.

\section{PLSR modeling for chemical composition}

Based on the optimized spectra and sample subsets, 10 MPA-optimized PLSR models were developed for soluble sugars, cellulose, hemicellulose, lignin, and ash. In order to conduct a fair comparison, five PLSR models were developed using full spectra and served as controls. During the developmental process, the numbers of samples for calibration and validation subsets were 
further reduced by the removal of sample outliers using Chauvenet's criterion [35]. To enhance the robustness of multivariate calibrations, all PLSR models were fully cross-validated using the "leave-one-out" method, where a single observation selected from calibration sample subsets was used as the validation data for each cross-validated process $[17,23]$. The optimal number of principal components (PCs) for each model was determined using root mean standard error of calibration (RMSEC) and the root mean standard error of cross-validation (RMSECV). In this case, 4 to 10 optimal PCs were obtained for 15 PLSR models accounting for over $96 \%$ of the variance, which further reduced the danger of over-fitting (Fig. 5a). Interestingly, the PCs of RF-optimized PLSR models generally accounted for higher values of variance than did CARS-optimized PLSR models and controls.

Summary statistics for the PLSR calibrations of chemical composition are provided in Table 1 . In general, all the chemical components were successfully predicted by
PLSR models and their uncertainties approximated those observed for wet chemical measurements, as indicated by low RMSEC (0.20-2.14) and RMSECV (0.27-2.78) values obtained. As a result, the relative high coefficients of determination of calibration $\left(R_{\mathrm{C}}^{2}\right)$ and cross-validations $\left(R_{\mathrm{CV}}^{2}\right)$ were obtained within the ranges of $0.79-0.98$ and 0.63-0.97, respectively. Significantly, both types of MPAoptimized PLSR calibration models generally displayed higher stability than did the controls, which exhibited relatively low RMSEC and RMSECV and high $R^{2}$ and $R^{2}$. Consequently, these results indicate that MPA could significantly improve the robustness of PLSR calibrations.

In the present study, fair prediction using externally validated samples was conducted to evaluate calibration equations. Summary statistics for the prediction of soluble sugars, cellulose, hemicellulose, lignin, and ash are provided in Table 2. In general, RF-optimized PLSR models performed better or similar as CARS-optimized models and controls when comparing values of the root mean

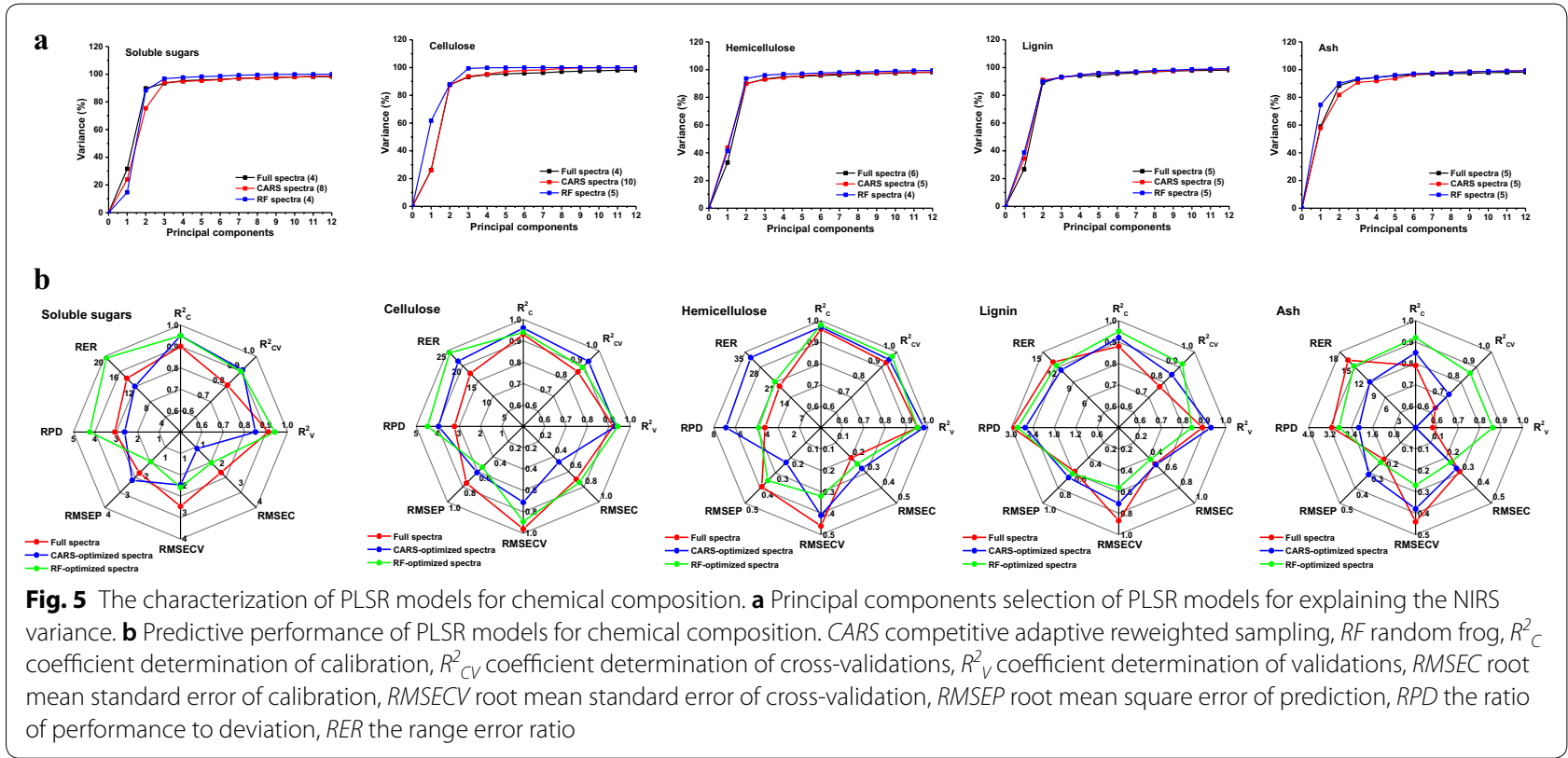

Table 1 Summary statistics for PLSR calibration models for chemical components

\begin{tabular}{|c|c|c|c|c|c|c|c|c|c|c|c|c|c|}
\hline \multirow[t]{2}{*}{ Parameter } & \multirow[t]{2}{*}{$N$} & \multicolumn{4}{|c|}{ Full spectra } & \multicolumn{4}{|c|}{ CARS-optimized spectra } & \multicolumn{4}{|c|}{ RF-optimized spectra } \\
\hline & & RMSEC & $R_{\mathrm{C}}^{2}$ & RMSECV & $R_{\mathrm{CV}}^{2}$ & RMSEC & $R^{2}{ }_{\mathrm{C}}$ & RMSECV & $R_{\mathrm{CV}}^{2}$ & RMSEC & $R^{2}{ }_{C}$ & RMSECV & $R_{\mathrm{CV}}^{2}$ \\
\hline Soluble sugars & 40 & 2.14 & 0.90 & 2.78 & 0.81 & 0.88 & 0.95 & 1.98 & 0.91 & 1.63 & 0.95 & 2.07 & 0.90 \\
\hline Cellulose & 45 & 0.70 & 0.93 & 0.96 & 0.86 & 0.47 & 0.96 & 0.71 & 0.93 & 0.74 & 0.94 & 0.89 & 0.89 \\
\hline Hemicellulose & 45 & 0.20 & 0.96 & 0.46 & 0.93 & 0.27 & 0.97 & 0.41 & 0.95 & 0.24 & 0.98 & 0.32 & 0.97 \\
\hline Lignin & 45 & 0.48 & 0.88 & 0.87 & 0.77 & 0.49 & 0.92 & 0.71 & 0.85 & 0.42 & 0.95 & 0.56 & 0.92 \\
\hline Ash & 45 & 0.29 & 0.79 & 0.44 & 0.63 & 0.27 & 0.85 & 0.38 & 0.72 & 0.23 & 0.92 & 0.27 & 0.86 \\
\hline
\end{tabular}

$N$ number of samples, CARS competitive adaptive reweighted sampling, $R F$ random frog, $R M S E C$ root mean standard error of calibration, $R^{2}{ }_{C}$ coefficient determination of calibration, $R M S E C V$ root mean standard error of cross-validation, $R_{C V}^{2}$ coefficient determination of cross-validation 
Table 2 Summary statistics for external validation of PLSR calibration models for chemical components

\begin{tabular}{|c|c|c|c|c|c|c|c|c|c|c|c|c|c|}
\hline \multirow[t]{2}{*}{ Parameter } & \multirow[t]{2}{*}{$N$} & \multicolumn{4}{|c|}{ Full spectra } & \multicolumn{4}{|c|}{ CARS-optimized spectra } & \multicolumn{4}{|c|}{ RF-optimized spectra } \\
\hline & & RMSEP & $R_{v}^{2}$ & RPD & RER & RMSEP & $R_{\mathrm{v}}^{2}$ & RPD & RER & RMSEP & $R_{v}^{2}$ & RPD & RER \\
\hline Soluble sugars & 13 & 2.17 & 0.91 & 3.07 & 14.18 & 2.55 & 0.85 & 2.61 & 12.07 & 1.57 & 0.94 & 4.24 & 19.60 \\
\hline Cellulose & 14 & 0.75 & 0.92 & 3.21 & 17.49 & 0.61 & 0.93 & 3.95 & 21.51 & 0.54 & 0.94 & 4.46 & 24.29 \\
\hline Hemicellulose & 14 & 0.39 & 0.95 & 4.18 & 19.12 & 0.23 & 0.98 & 7.08 & 32.42 & 0.35 & 0.95 & 4.65 & 21.31 \\
\hline Lignin & 14 & 0.58 & 0.89 & 2.98 & 12.97 & 0.66 & 0.93 & 2.61 & 11.40 & 0.61 & 0.84 & 2.83 & 12.33 \\
\hline Ash & 14 & 0.21 & 0.58 & 3.13 & 16.05 & 0.31 & 0.08 & 2.12 & 10.87 & 0.23 & 0.86 & 2.86 & 14.65 \\
\hline
\end{tabular}

$N$ number of samples, CARS competitive adaptive reweighted sampling, $R F$ random frog, $R M S E P$ root mean square error of prediction, $R^{2}{ }_{V}$ coefficient determination of validation, $R P D$ the ratio of performance to deviation, $R E R$ the range error ratio

square error of prediction (RMSEP) and $R_{\mathrm{V}}^{2}$ for prediction of soluble sugars, cellulose, and lignin. On the contrary, CARS optimization could significantly improve the performance of PLSR multivariate calibrations for hemicellulose prediction. In recent years, numerous studies have reported that ash level is not easily or directly measurable by NIRS, due to its inorganic nature $[17,18,24$, 36]. Similarly, the statistics of CARS-optimized PLSR models and controls suggest fair to the poor prediction for ash in the present study, rendering these two methods suitable only for very rough screening. However, inorganic ash was successfully predicted by RF-optimized PLSR models with significantly higher $R_{\mathrm{V}}^{2}$ and lower RMSEP.

Recent studies suggest that for research calibrations, excellent calibration models must exhibit a ratio of performance to deviation (RPD) and range error ratio (RER) values greater than 3 and 15 , respectively [22-25]. In this study, almost all RPD and RER values of RF-optimized PLSR models were higher or similar to standard values (Table 2), resulting in relatively better correlations between predicted and reference values for chemical components (Additional file 1: Fig. S3). In order to achieve greater visual comprehension, the spider diagram was adopted for comparing the predictive performance of employed PLSR models (Fig. 5b). It was obvious that RF was a more efficient algorithm than CARS for improving the predictive performance of PLS multivariate calibrations. Taken together, these results indicate that MPA optimization shows promise for improving the accuracy and robustness of PLS multivariate calibrations and that RF-optimized PLSR models are adequate for the determination of JA chemical composition.

\section{NIRS modeling for biomass digestibility}

To assess biomass digestibility, six similarly optimized MPA-optimized PLSR models were developed for JA accessions obtained nationwide, while three PLSR models served as controls. Similarly, RF-optimized PLSR models generally produced higher values of variance than did
CARS-optimized PLSR models and controls at optimal PCs (Fig. 6a). Table 3 provides an overview of nine PLSR calibrations for the prediction of hexoses, pentoses, and total carbohydrates. In general, the prediction models of biomass digestibility exhibited a slight decrease in $R^{2}{ }_{\mathrm{C}}$ values $(0.68-0.93)$ and $R_{C V}^{2}$ values $(0.48-0.87)$ than did prediction models of chemical composition. However, six MPA-optimized PLSR models for biomass digestibility showed significantly higher $R_{\mathrm{C}}^{2}(0.86-0.93)$ and $R_{\mathrm{CV}}^{2}$ (0.75-0.87) values than corresponding controls. Meanwhile, extremely low RMSEC (1.05-1.55) and RMSECV (1.85-2.11) values were also obtained using MPA-optimized calibrations, indicating superior stability.

Subsequently, lower RMSEP (1.24-2.55), higher RPD (1.74-3.96), and RER (8.38-20.96) values were obtained using robust MPA-optimized calibrations (Table 4). In particular, the 14 externally validated samples exhibited strong correlations between predicted and reference values (Additional file 1: Fig. S3B, C). By contrast, the controls demonstrated very poor predictive capacity (Table 4 and Additional file 1: Fig. S3A). These results demonstrate that MPA could clearly improve upon prediction performance accuracies of the PLSR model for biomass digestibility prediction, which verified our previously stated results that CARS and RF displayed superior efficiency for the selection of both informative variables and elimination of uninformative variables (Fig. 4). Notably, comparison of MPA algorithms indicated that RF was more efficient than CARS in improving the performance of PLSR calibrations for predicting yields of hexoses, pentoses, and total carbohydrates released after pretreatment followed by enzymatic hydrolysis (Fig. 6b). Together these results provide important insights into how the optimization of spectral variable selection by MPA could significantly enhance both the stability and accuracy of PLSR models for chemical composition and biomass digestibility. Such models form the basis of a precise and efficient methodology for predicting both chemical composition and biomass digestibility of JA feedstock. 

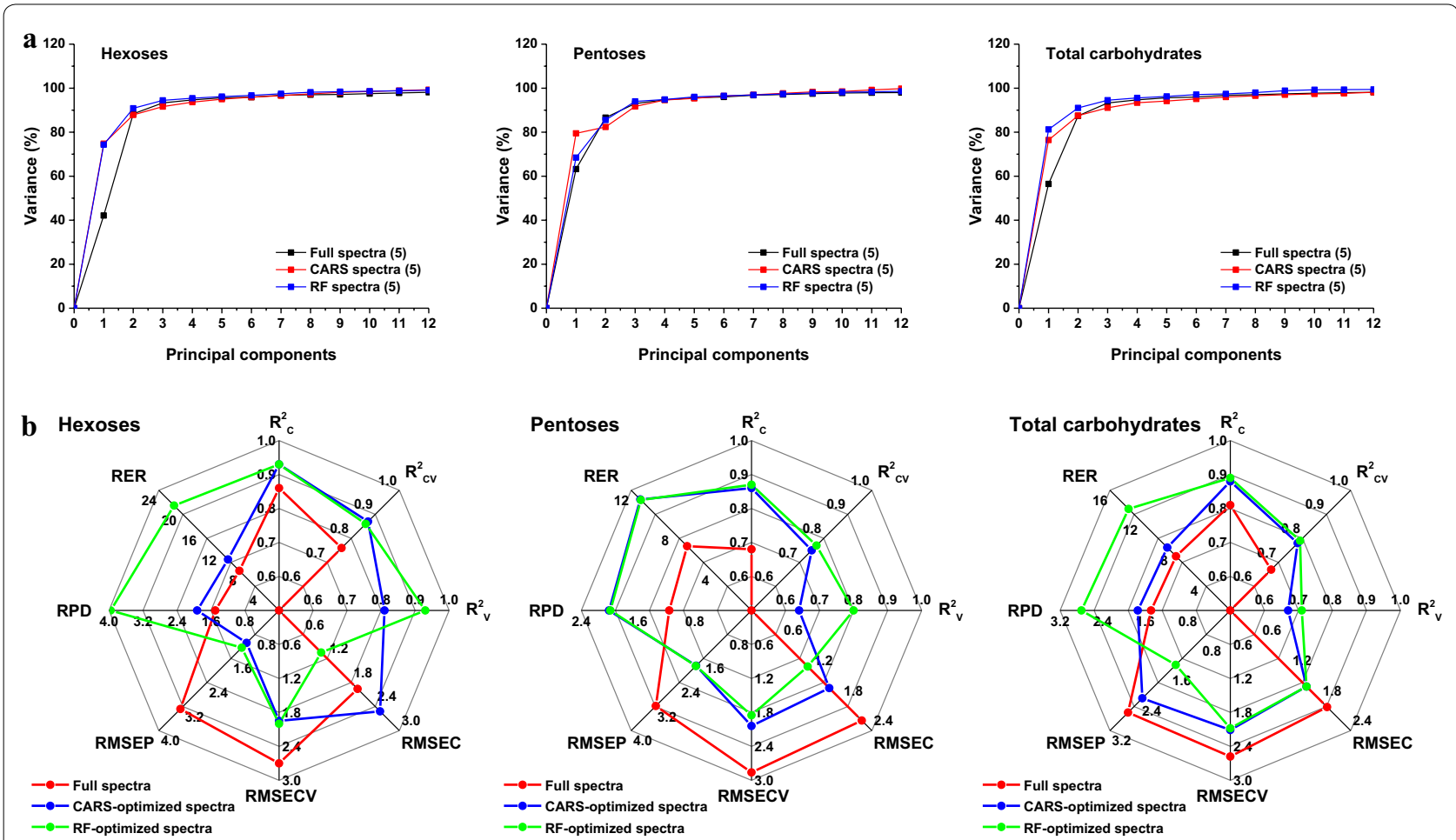

Fig. 6 The characterization of PLSR models for biomass digestibility. a Principal components selection of PLSR models for explaining the NIRS variance. $\mathbf{b}$ Predictive performance of PLSR models for biomass digestibility. CARS competitive adaptive reweighted sampling, $R F$ random frog, $R^{2} C$ coefficient determination of calibration, $R^{2}{ }_{C V}$ coefficient determination of cross-validations, $R^{2}{ }_{V}$ coefficient determination of validations, $R M S E C$ root mean standard error of calibration, RMSECV root mean standard error of cross-validation, RMSEP root mean square error of prediction, RPD the ratio of performance to deviation, RER the range error ratio

Table 3 Summary statistics for PLSR calibration models for biomass digestibility

\begin{tabular}{|c|c|c|c|c|c|c|c|c|c|c|c|c|c|}
\hline \multirow[t]{2}{*}{ Parameter } & \multirow[t]{2}{*}{$N$} & \multicolumn{4}{|c|}{ Full spectra } & \multicolumn{4}{|c|}{ CARS-optimized spectra } & \multicolumn{4}{|c|}{ RF-optimized spectra } \\
\hline & & RMSEC & $R_{\mathrm{C}}^{2}$ & RMSECV & $R_{\mathrm{CV}}^{2}$ & RMSEC & $R_{\mathrm{C}}^{2}$ & RMSECV & $\overline{R_{\mathrm{cv}}^{2}}$ & RMSEC & $R^{2}{ }_{\mathrm{C}}$ & RMSECV & $R_{\mathrm{CV}}^{2}$ \\
\hline Hexoses & 45 & 1.96 & 0.86 & 2.70 & 0.76 & 1.29 & 0.93 & 1.97 & 0.87 & 1.05 & 0.93 & 2.00 & 0.86 \\
\hline Pentoses & 45 & 2.20 & 0.68 & 2.86 & 0.48 & 1.55 & 0.86 & 2.04 & 0.75 & 1.12 & 0.87 & 1.85 & 0.77 \\
\hline Total & 45 & 1.93 & 0.81 & 2.58 & 0.67 & 1.52 & 0.88 & 2.11 & 0.78 & 1.52 & 0.89 & 2.08 & 0.79 \\
\hline
\end{tabular}

$N$ number of samples, CARS competitive adaptive reweighted sampling, $R F$ random frog, $R M S E C$ root mean standard error of calibration, $R^{2}{ }_{C}$ coefficient determination of calibration, $R M S E C V$ root mean standard error of cross-validation, $R_{C V}^{2}$ coefficient determination of cross-validation

Table 4 Summary statistics for external validation of PLSR calibration models for biomass digestibility

\begin{tabular}{|c|c|c|c|c|c|c|c|c|c|c|c|c|c|}
\hline \multirow[t]{2}{*}{ Parameter } & \multirow[t]{2}{*}{$N$} & \multicolumn{4}{|c|}{ Full spectra } & \multicolumn{4}{|c|}{ CARS-optimized spectra } & \multicolumn{4}{|c|}{ RF-optimized spectra } \\
\hline & & RMSEP & $R_{\mathrm{v}}^{2}$ & RPD & $\overline{\text { RER }}$ & RMSEP & $R_{\mathrm{v}}^{2}$ & RPD & $\overline{\text { RER }}$ & RMSEP & $R_{\mathrm{v}}^{2}$ & RPD & RER \\
\hline Hexoses & 14 & 3.28 & 0.44 & 1.50 & 7.92 & 2.55 & 0.81 & 1.93 & 10.19 & 1.24 & 0.93 & 3.96 & 20.96 \\
\hline Pentoses & 14 & 3.18 & 0.15 & 1.16 & 6.42 & 1.84 & 0.64 & 2.01 & 11.09 & 1.85 & 0.80 & 1.99 & 11.03 \\
\hline Total & 14 & 2.72 & 0.35 & 1.49 & 7.21 & 2.34 & 0.67 & 1.74 & 8.38 & 1.45 & 0.71 & 2.80 & 13.52 \\
\hline
\end{tabular}

$N$ number of samples, CARS competitive adaptive reweighted sampling, $R F$ random frog, $R M S E P$ root mean square error of prediction, $R^{2}{ }_{V}$ coefficient determination of validation, $R P D$ the ratio of performance to deviation, $R E R$ the range error ratio 


\section{Comprehensive assessment of feedstock quality score}

Based on chemical composition and biomass digestibility (total carbohydrates released after pretreatment and subsequent enzymatic hydrolysis), the feedstock quality of tested JA accessions was comprehensively evaluated using the GRA model. As shown in Fig. 7a, solid lines represent the normal distribution and are intended to highlight any discrepancy between the histogram and normality. With regard to FQS, frequency refers to the number of samples within a given range and the percentage of each FQG is indicated above the histograms. In general, the FQS distribution was similar to a normal distribution, which is not unexpected for a nationwide feedstock population. Moreover, the mean value of FQS was 28.6 and majority of the samples fell within D (43.1\%) and $\mathrm{E}(39.7 \%)$ grades. By contrast, only $3.4 \%$ and $5.2 \%$ of JA samples were assigned to A and B grades (details could be found in Additional file 1: Table S1). Hence, there is a growing need to focus efforts on JA germplasm resource selection, breeding, and genetic modification to improve biomass feedstock quality for efficient bioethanol production.

Notably, the JA accessions obtained from six areas of China displayed very diverse FQS distributions (Fig. 7b) and mean FQS values were ranked in the order of NEC $(53.5)>$ SWC $\quad(35.3)>\mathrm{CSC} \quad(30.1)>\mathrm{NWC} \quad(24.3)>\mathrm{NC}$
SW (23.6) $>$ EC (23.3). ANOVA analysis showed that JA accessions from NEC exhibited significantly higher FQS than those from NC, EC, and NWC $(P<0.001)$. However, high-quality JA accessions could be found in most sampled regions (except for EC). Summary statistics of chemical components, biomass digestibility, and FQS of JA accessions assigned to grade $\mathrm{A}$ and $\mathrm{B}$ are detailed in Table 5. As expected, each selected JA accession possessed a high level of soluble sugars, cellulose, and hemicellulose, low levels of lignin and ash, and outstandingly high biomass digestibility. Therefore, these accessions are currently the most ideal JA feedstocks for bioethanol production in China, demonstrating that the GRA was adequate for high-throughput biomass feedstock quality evaluation.

\section{Qualitative analysis of feedstock quality grade}

For the purposes of the industrial application, further research should focus on rapid and precise classification of a large number of biomass feedstocks into different FQGs to achieve commoditization of lignocellulosic biofuel. In this work, KS algorithm-partitioned sample subsets coupled to both CARS and RF algorithms were employed to develop two optimized PLS-DA models for FQG classification of JA stem biomass, while PLS-DA models based on full spectra served as controls. Summary statistics of three PLS-DA models for qualitative analysis of FQG are presented in Table 6. Notably, the FQG values of JA stem were successfully classified using PLS-DA models to obtain relatively higher values of the multiple coefficients of determination $\left(R^{2}: 0.76-0.96\right)$ and the explained variation in the test set $\left(Q^{2}: 0.52-0.83\right)$. In addition, it was obvious that two MPA algorithms could significantly enhance the stability of PLS-DA models. Regarding classification accuracy, the highest correct rates, $97.8 \%$ in the calibration subset and $91.1 \%$ in the validation subset, were obtained using the robust RFoptimized PLS-DA model, which were superior to rates
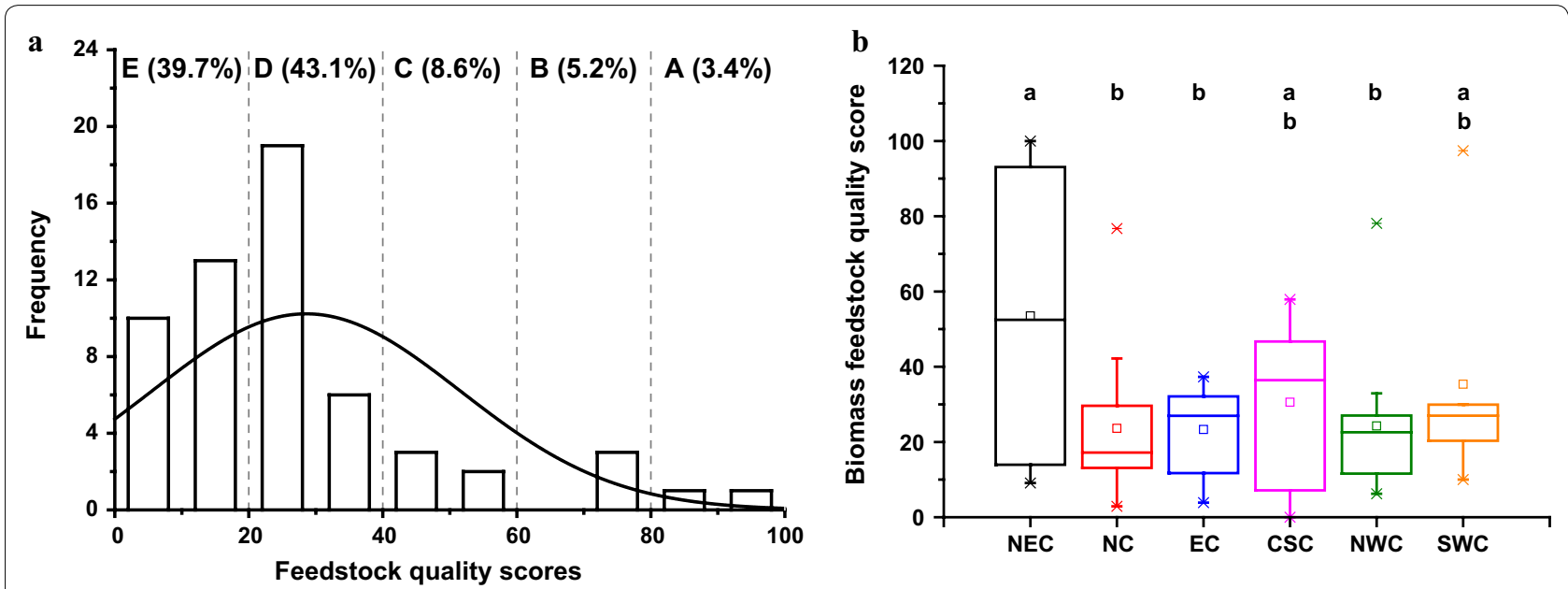

Fig. 7 Feedstock quality grades distribution of 59 Jerusalem artichoke accessions in China. a National scope. b Regional scope. Different small letters indicate significant differences among six typical regions at $P<0.001$. The solid lines overlaid upon each histogram represent normal distributions and were used to embody the discrepancy between histogram and normality. NEC Northeast China, NC North China, EC East China, CSC Central-South China, NWC Northwest China, and SWC Southwest China 
Table 5 The statistics of Jerusalem artichoke accessions ideal for bioethanol production in China

\begin{tabular}{|c|c|c|c|c|c|c|c|}
\hline Parameter & E025 & E047 & E026 & E058 & E053 & E044 & E059 \\
\hline Soluble sugars (\% dry matter) & 15.4 & 32.1 & 13.1 & 23.4 & 26.4 & 30.1 & 21.8 \\
\hline Cellulose (\% dry matter) & 30.9 & 22.5 & 31.6 & 24.8 & 24.3 & 23.2 & 27.5 \\
\hline Hemicellulose (\% dry matter) & 18.4 & 11.4 & 18.8 & 12.0 & 11.5 & 11.4 & 13.4 \\
\hline Lignin (\% dry matter) & 21.1 & 13.6 & 20.9 & 14.3 & 14.5 & 14.2 & 14.8 \\
\hline Ash (\% dry matter) & 3.4 & 3.7 & 3.0 & 5.2 & 4.9 & 3.9 & 4.5 \\
\hline $\begin{array}{l}\text { Biomass digestibility (\% total carbohy- } \\
\text { drates) }\end{array}$ & 79.2 & 82.8 & 77.3 & 78.4 & 78.5 & 78.8 & 76.1 \\
\hline Region & NEC & SWC & NEC & NWC & $\mathrm{NC}$ & SWC & $\mathrm{CSC}$ \\
\hline Feedstock quality score & 100.0 & 97.4 & 86.2 & 78.1 & 76.7 & 70.7 & 60.1 \\
\hline Feedstock quality grade & $A$ & $A$ & $A$ & $\mathrm{~B}$ & $\mathrm{~B}$ & $\mathrm{~B}$ & $\mathrm{~B}$ \\
\hline
\end{tabular}

NEC northeast China, SWC southwest China, NWC northwest China, NC north China, CSC central-south China

Table 6 Summary statistics of PLS-DA models for qualitative analysis of biomass feedstock quality grades

\begin{tabular}{|c|c|c|c|c|c|c|c|}
\hline \multirow[t]{2}{*}{ Parameter } & \multirow[t]{2}{*}{ PCs } & \multirow[t]{2}{*}{$R^{2}$} & \multirow[t]{2}{*}{$Q^{2}$} & \multicolumn{2}{|c|}{ Calibration } & \multicolumn{2}{|c|}{ Validation } \\
\hline & & & & $N$ & Correct (\%) & $N$ & Correct (\%) \\
\hline Full spectra & 4 & 0.76 & 0.52 & 45 & 97.8 & 14 & 86.7 \\
\hline CARS-optimized spectra & 7 & 0.96 & 0.83 & 45 & 95.6 & 14 & 88.9 \\
\hline RF-optimized spectra & 3 & 0.91 & 0.80 & 45 & 97.8 & 14 & 91.1 \\
\hline
\end{tabular}

$P C s$ principal components, $R^{2}$ the multiple coefficients of determination, $Q^{2}$ the explained variation in the test set, $N$ number of samples

obtained using the CARS-optimized PLS-DA model. Therefore, the RF algorithm was more efficient than the CARS algorithm for improving the predictive performance of PLS multivariate calibrations. Overall, these results indicate that the optimization of spectral variable selection by MPA could significantly enhance the stability and classification accuracy of PLS-DA multivariate calibrations, consistent with the quantitative determinations of JA stem chemical composition and biomass digestibility.

\section{Discussion}

Evaluation and selection of ideal feedstock among bioenergy crops are necessary to enhance lignocellulosic biofuel production [37, 38]. Recent developments in biochemical conversion have demonstrated that the chemical and physical properties of lignocellulose are crucial indicators of biomass feedstock quality [12]. In terms of chemical composition, soluble sugars, cellulose, and hemicellulose act as primary resources for biofuel production, while lignin and ash are considered useless components or barriers to biochemical conversion [39]. Therefore, inherent variability in chemical composition fundamentally determines the theoretical energy potential of lignocellulosic feedstock. With respect to physical structure, lignocellulose is a compact dimensional molecule, which gives rise to biomass recalcitrance such that deconstruction of any of physical properties is constrained by the other properties. Recently, results of a combined pretreatment and saccharification assay have increased our understanding of phenomena that include "biomass reactivity" or "biomass digestibility," properties which could be used to directly predict usability of lignocellulosic feedstock [32]. Therefore, the current knowledge suggests that chemical composition and biomass digestibility should be considered together during lignocellulosic biomass evaluation and selection. Prior to this study, few reports have described the comprehensive assessment of lignocellulosic biomass based on both chemical composition and biomass digestibility $[32,37,38,40,41]$. In addition, due to innate variability in chemical composition and biomass digestibility, it is critical to understand that not all lignocellulosic biomass is suitable for conversion into biofuels and biochemicals [14]. Therefore, particular attention should be paid to ideal feedstock selection when JA stem biomass was concerned.

In the current study, we determined the variability in chemical composition and biomass digestibility of sugarrich stem biomass of 59 JA clones collected nationwide. These JA accessions were uniformly distributed within longitudes between 82.06 and 126.75 and latitudes between 25.60 and 44.86 and exhibited diverse genotypes and phenotypes [7-9]. Notably, innate JA feedstock 
quality variability based on chemical composition and biomass digestibility was identified at both national and regional levels (Fig. 2), which could be caused by the interaction between diverse JA genotypes and their various environments. These results suggest that a potential exists for selection of ideal varieties to enhance biofuel conversion or bioenergy crop breeding and genetic modification. Besides, ideal bioethanol feedstock could be identified by high levels of carbohydrates (soluble sugars, cellulose, and hemicellulose), high biomass digestibility and low level of waste components (lignin and ash) [14, 17]. For the purpose of screening the ideal bioethanol feedstock, we transformed the feedstock quality properties to a fair FQS and further classified it into five FQG levels. As a result, 7 clones from 6 sampled regions were deemed superior biomass feedstocks for bioethanol production. Clearly, these results demonstrate the high potential of JA to serve as an ideal bioenergy crop for nationwide cultivation. Although the JA accessions in this study were highly representative, these results should be interpreted with caution due to the limited size of the sample population. Therefore, attention should be paid to the continued collection and identification of germplasm resources.

With the development of computational science, NIRS coupled with PLS has gained extensive popularity for the qualitative and quantitative analysis of chemical and physical properties of biomass feedstock due to its highthroughput, low-cost, and non-destructive nature [17, 19, $20,22-24,33,36]$. However, the intrinsically disadvantage of PLS was that it requires a crucial process to build a credible model for given chemical or biological data, which is known as spectral variable selection [23]. In this sector, MPA has offered great advantages by statistically analyzing the distribution of an interested outcome of the sub-models derived with the aid of Monte Carlo Sampling, which could significantly improve the accuracy and robustness of prediction models [26, 28]. In this study, two novel MPA algorithms based on different theory (CARS and RF) were employed to establish a series of optimal spectral variable subsets derived from full NIRS data. One interesting finding was that only $17-82$ and 15-31 spectral variables were selected by CARS and RF from 1557 variables within full spectra, respectively. This finding indicates that MPA could judiciously identify most of the informative spectral variables while eliminating the uninformative spectral variables $[23,29$, 30]. Another important finding was that several variables were collectively selected by both of these algorithms for each predictive indictor, providing very important information that could be used for further improve prediction of biomass chemical and physical properties [23]. Based on these optimized spectral variable subsets, a series of MPA-optimized PLSR models and MPA-optimized PLS-DA models were developed for quantitative and qualitative analysis of JA biomass feedstocks. The results indicated that RF was better for improving both qualitative and quantitative PLS models than CARS (Figs. 5, 6 and Table 6). In comparison with the NIR models of previous studies, RF-optimized PLSR models exhibited a better performance for the prediction of chemical composition (Additional file 1: Table S2) and biomass digestibility (Additional file 1: Table S3) with more reasonable values of RMSEC, $R_{C}^{2}$, RMSECV, $R_{C V}^{2}$, RMSEP, $R_{V}^{2}$, RPD, and RER [17, 19, 22, 23, 42]. Obviously, spectral variable selection using MPA could significantly improve the predictive performance of PLS multivariate calibration in the qualitative and quantitative analysis of biomass feedstock.

\section{Conclusions}

In this study, 59 JA clone stems originating from six regions of China exhibited diverse chemical compositions, biomass digestibility, and variable NIRS results, which were applicable for statistical analysis and NIRS modeling. Soluble sugars, cellulose, hemicellulose, lignin, ash, and released hexoses, pentoses, and total carbohydrates were then successfully predicted via MPA-optimized PLSR models. Based on the reliable and accurate data of chemical composition and biomass digestibility, all 59 JA accessions were comprehensively evaluated for FQS by GRA and assigned into five FQGs. Notably, seven clones were identified as the most ideal JA feedstocks for bioethanol production in China. Finally, the JA accessions studied in this work were rapidly and successfully classified using a MPA-optimized PLS-DA model. In conclusion, this study provides a practical strategy of high-throughput screening lignocellulosic biomass for bioethanol production.

\section{Methods}

\section{Sample collection and preparation}

A total of 59 JA natural clones were collected nationwide from 2006 to 2012. JA stem samples were harvested on their dates of physiological maturity and were processed at the China Agricultural University Zhuozhou Experimental Station $\left(39^{\circ} 47^{\prime} \mathrm{N}, 115^{\circ} 87^{\prime} \mathrm{E}\right)$ in Hebei Province in 2014. Stem samples were handled according to the protocols developed by the National Renewable Energy Laboratory/TP-510-42620 [43]. First, samples were ground using a crusher mill into particles $1-2 \mathrm{~cm}$ in size and dried at $45{ }^{\circ} \mathrm{C}$ for $48 \mathrm{~h}$ after heat at $105{ }^{\circ} \mathrm{C}$ for $20 \mathrm{~min}$. Next, the dried particles were ground into powder and passed through a combined $-40 /+80$ mesh screen. Finally, the mesh-screened samples (as dry matter) were stored in a dry container until use. A spatial distribution 
map of collection sites of JA accessions was generated using ArcGIS 10.3.

\section{Biomass components and digestibility analysis}

The main topic of this research was to build upon the laboratory-scale alkali-based conversion process developed by Li et al. [11] to develop a comprehensive and highthroughput lignocellulosic biomass feedstock screening system for bioethanol production. Soluble sugars were extracted from dried JA stem samples using distilled water and quantified using the anthrone-sulfuric acid method using a UV-VIS spectrometer (TU-1901, Beijing Purkinje General Instrument Co., Ltd., Beijing, China). A standard curve was plotted using D-glucose as the standard (Xilong Scientific Co., Ltd., China). Ash content was determined using a muffle furnace (VULCAN 3-550, Densply International, Inc., York, PA, USA) with $30 \mathrm{~mL}$ ceramic crucibles according to LAP NREL/TP-510-42622 [44]. Structural carbohydrates and lignin were extracted using a two-step sulfuric acid hydrolysis process with dried JA stem samples according to NREL/TP-510-42618, NREL/TP-510-42619, and NREL/TP-510-42621 with minor modifications [45-47]. Structural carbohydrates (i.e., glucose, xylose, and arabinose) were measured using an HPLC system (1260 series, Agilent Technologies, Santa Clara, CA, USA) equipped with an Aminex HPX$87 \mathrm{H}$ chromatography column $(300 \mathrm{~mm} \times 7.8 \mathrm{~mm}$, particle size $9 \mu \mathrm{m}$, Bio-Rad Laboratories, Hercules, CA, USA). Lignin content was determined using a UV-VIS spectrometer (TU-1901, Beijing Purkinje General Instrument Co., Ltd.) and the same muffle furnace described above. Calculations of cellulose, hemicellulose, and lignin content were performed according to Li et al. [11]. Cellulose was calculated from glucose content, while hemicellulose was calculated from the sum of xylose and arabinose content values. Lignin was calculated from the sum of acidsoluble lignin and acid-insoluble lignin content values. All experiments were carried out in triplicate.

Biomass digestibility was defined by accounting for the hexoses, pentoses, and total carbohydrates released from the soluble sugars extracted biomass feedstock after alkali-based pretreatment followed by enzymatic hydrolysis [11]. For pretreatment, a 2-g quantity of soluble sugars-extracted JA sample was mixed with $40 \mathrm{~mL} 2 \%(\mathrm{w} / \mathrm{v})$ sodium hydroxide and shaken at $150 \mathrm{rpm}$ for $2 \mathrm{~h}$ at $50{ }^{\circ} \mathrm{C}$. Next, each pretreated sample was washed five times with $20 \mathrm{~mL}$ distilled water and dried at $80{ }^{\circ} \mathrm{C}$ for subsequent enzymatic hydrolysis. During enzymatic hydrolysis, each pretreated sample was mixed with $0.2 \%(\mathrm{w} / \mathrm{v})$ mixed-cellulases containing $\beta$-glucanase $\left(\geq 3.6 \times 10^{4} \mathrm{U}\right)$, cellulase $\left(\geq 360 \times 10^{2} \mathrm{U}\right)$, and xylanase $\geq 6 \times 10^{4} \mathrm{U}$ from Imperial Jade Biotechnology Co., Ltd.) in a volume of $40 \mathrm{~mL}$ in a $50-\mathrm{mL}$ centrifuge tube and shaken at $150 \mathrm{rpm}$ at $50{ }^{\circ} \mathrm{C}$ for $48 \mathrm{~h}$. Glucose and xylose released after alkali-based pretreatment followed by enzymatic hydrolysis were determined by HPLC as described above. Statistical and variance (ANOVA) analysis was calculated using IBM SPSS Statistics software (ver. 24). All experiments were carried out in triplicate.

\section{Near-infrared spectroscopy pretreatments}

JA stem dry matter was scanned and recorded in triplicate using a Thermo Antaris II FT-NIR Analyzer (Thermo Scientific, Inc., Madison, WI, USA) equipped with a diffuse reflectance accessory device. Each spectrum was averaged over 64 scans at a resolution of $4 \mathrm{~cm}^{-1}$ within the wavenumber range of $4000-10,000 \mathrm{~cm}^{-1}$ at room temperature (Additional file 2). Spectrometer control and data collection were conducted using TQ Analyst software (ver. 9.3). In order to correct spectra scatter, all spectra were first adjusted using multiplicative scatter correction. Next, the Savitzky-Golay smoothing filter and the first derivative were employed to reduce random noise and to resolve spectral peak overlap and eliminate linear baseline drift [23]. The purpose of the aforementioned corrections was to remove multiplicative and additive effects stemming from instrument settings or variations caused by the sample and environmental conditions [19]. After pretreatments, six principal component analysis models were developed using TQ Analyst software (ver. 9.3) to generate a 3D NIRS scatter plot [33].

\section{The development of MPA-optimized PLSR models}

Based on pretreated sample spectra, both CARS and RF algorithms were applied to determine spectral variables sets using ChemDataSolution software (ver. 2.0). For a fair multivariate prediction, one of every five samples was sorted into validation sets using $\mathrm{KS}$ algorithms based on full spectra and two types of characteristic spectra; the remaining samples were used for generating calibration sets. Calibration and validation sets were compared in terms of both their chemical components and biomass digestibility. In addition, the NIRS plots distribution of calibration and validation sets was also compared using eighteen principal component analysis models in the present study. Based on the MPA-optimized spectra, sixteen PLSR multivariate calibrations were developed to predict soluble sugars, cellulose, hemicellulose, lignin, ash, and biomass digestibility (hexoses, pentoses, and total carbohydrates) using ChemDataSolution software (ver. 2.0); eight PLSR models based on full spectra served as controls. To select the optimum number of factors and to avoid over-fitting, the "leave-one-out" method was used for cross-validation while developing PLSR models [17, 19]. On the one hand, the robustness of PLSR models was evaluated using several chemometrics parameters, 
including RMSEC, RMSECV and $R_{C}^{2}$, and $R^{2}{ }_{\mathrm{CV}}$. On the other hand, the accuracy of each multivariate calibration model could be determined by RMSEP and $R_{\mathrm{V}}^{2}$. Finally, $R P D$ and RER were employed to ascertain MPA enhancement of prediction performance.

\section{Grey relational grade analysis}

In this study, JA stem feedstock quality specifications could be divided into two categories: chemical components (soluble sugars, cellulose, hemicellulose, lignin, and ash) and biomass digestibility (hexoses, pentoses, and total carbohydrates). These quality specifications were used to calculate and compare the FQS values of JA samples collected from different geographic regions. To address this challenge, a high-efficiency evaluation and selection model was developed based on grey relational grade analysis theory, which is an essential component of the grey system theory formulated by Julong Deng [16]. Both chemical components and biomass digestibility (total carbohydrates released from pretreatment and subsequent enzymatic hydrolysis) were assigned the same weight of 0.5 in this study. To meet the demands of industry, the FQS values of JA samples were assigned to five grade levels and ranked in the order A $(80-100) \geq B$ $(60-80) \geq C \quad(40-60) \geq D(20-40) \geq E(0-20)$. The GRA was processed using MATLAB software (ver. 2012b) and the procedure code is documented in Additional file 3.

\section{The development of MPA-optimized PLS-DA models}

Based on full spectra and MPA-optimized spectra, three PLS-DA multivariate calibrations were developed for FQG classification using ChemDataSolution software (ver. 2.0). One of every five samples was sorted into validation sets using KS algorithms based on full spectra and two characteristic spectra and the remaining samples were used for the calibration sets. In addition, the multiple coefficients of determination $\left(R^{2}\right)$, the explained variation in the test set $\left(Q^{2}\right)$, and the correct rate were employed to ascertain MPA enhancement of classification performance [21].

\section{Additional files}

\footnotetext{
Additional file 1: Fig. S1. Histograms of chemical components and biomass digestibility based on full spectra (A), CARS-optimized spectra (B), and RF-optimized spectra (C). The solid lines and dashed lines overlaid upon each histogram represent normal distributions and were used to embody the discrepancy between each histogram and normality. Fig. S2. PCA plots distribution of chemical components and biomass digestibility based on full spectra (A), CARS-optimized spectra (B), and RF-optimized spectra (C). Fig. S3. Plots of predicted versus reference values of PLSR models based on full spectra (A), CARS-optimized spectra (B), and RF-optimized spectra (C). $R^{2}$ v represents the square of the correlation coefficients of the external validation subsets. Table S1. Feedstock quality
}

grades of 59 Jerusalem artichoke accessions. Table S2. A summary of NIR application in different biomass feedstocks for chemical components. Table S3. A summary of NIR application in different biomass feedstocks for biomass digestibility.

Additional file 2. Raw NIRS data.

Additional file 3. The procedure code of grey relational grade analysis.

\section{Abbreviations}

JA: Jerusalem artichoke; NIRS: near-infrared spectroscopy; KS: KennardStone algorithm; PLSR: partial least squares regression; PLS-DA: partial least squares-discriminant analysis; PCA: principal component analysis; MPA: model population analysis; CARS: competitive adaptive reweighted sampling; RF: random frog; RMSEC: root mean standard error of calibration; RMSECV: root mean standard error of cross-validation; RMSEP: root mean square error of prediction; $R_{c}^{2}$ : coefficient determination of calibration; $R_{\text {cv: }}^{2}$ coefficient determination of cross-validations; $R^{2} v$ : coefficient determination of validations; RPD: the ratio of performance to deviation; RER: the range error ratio; GRA : grey relational grade analysis; FQS: feedstock quality score; FQGs: feedstock quality grades; $R^{2}$ : the multiple coefficient of determination; $Q^{2}$ : the explained variation in the test set.

\section{Authors' contributions}

GHX organized this research as the laboratory chief. ZL collected the JA germplasm resources. ML and JW conducted chemical composition and biomass digestibility determination. ML and SH designed the lignocellulosic biomass feedstocks screening system and built all the discussed models. ML performed the statistical analysis and drafted figures and tables. All authors read and approved the final manuscript.

\section{Author details}

${ }^{1}$ College of Agronomy and Biotechnology, China Agricultural University, Beijing 100193, China. ${ }^{2}$ National Energy R\&D Center for Non-food Biomass, China Agricultural University, Beijing 100193, China. ${ }^{3}$ Chinese Academy of Agricultural Engineering Planning and Design, Beijing 100125, China.

\section{Acknowledgements}

The authors would like to acknowledge Zhongdou Gao for JA accessions sampling, and Mingxing Xue, Lei Chen, and Yan Li for chemical composition and biomass digestibility determination. This work was supported by Department of Energy Conservation and Technology Equipment of China's National Energy Administration (Science and Technology Department, No. [2012] 32) and funded by China Datang New Energy Co. Ltd and Henan Tianguan Group Co., Ltd.

\section{Competing interests}

The authors declare that they have no competing interests.

\section{Availability of data and materials}

The datasets supporting the conclusions of this article are included within the article and its additional files.

Consent for publication

Not applicable.

Ethics approval and consent to participate

Not applicable.

\section{Publisher's Note}

Springer Nature remains neutral with regard to jurisdictional claims in published maps and institutional affiliations.

Received: 19 October 2018 Accepted: 10 December 2018

Published online: 19 December 2018 


\section{References}

1. Wang X, Yang L, Steinberger Y, Liu Z, Liao S, Xie G. Field crop residue estimate and availability for biofuel production in China. Renew Sustain Energy Rev. 2013;27:864-75

2. BP. BP statistical review of world energy. 2017: 3.

3. Demirbas A, Demirbas A. Modernization of biomass energy conversion facilities. Energy Sources Part B. 2007;2:227-35.

4. Zhang Y, Lin F, Wang X, Zou J, Liu S. Annual accounting of net greenhouse gas balance response to biochar addition in a coastal saline bioenergy cropping system in China. Soil Tillage Res. 2016;158:39-48.

5. Liu S, Zhao C, Zhang Y, Hu Z, Wang C, Zong Y, Zhang L, Zou J. Annual net greenhouse gas balance in a halophyte (Helianthus tuberosus) bioenergy cropping system under various soil practices in Southeast China. GCB Bioenergy. 2015;7:690-703.

6. Kim S, Kim CH. Evaluation of whole Jerusalem artichoke (Helianthus tuberosus L.) for consolidated bioprocessing ethanol production. Renew Energy. 2014;65:83-91.

7. Liu ZX, Spiertz JHJ, Sha J, Xue S, Xie GH. Growth and yield performance of Jerusalem artichoke clones in a semiarid region of china. Agron J. 2012:104:1538.

8. Liu ZX, Steinberger Y, Xu C, Wang JS, Xie GH. Chemical composition and potential ethanol yield of Jerusalem artichoke in a semi-arid region of China. Ital J Agron. 2015;10:34-43.

9. Liu ZX, Han LP, Steinberger Y, Xie GH. Genetic variation and yield performance of Jerusalem artichoke germplasm collected in China. Agric Sci China. 2011;10:668-78.

10. Matías J, González J, Royano L, Barrena RA. Analysis of sugars by liquid chromatography-mass spectrometry in Jerusalem artichoke tubers for bioethanol production optimization. Biomass Bioenergy. 2011;35:2006-12.

11. Li M, Wang J, Yang YZ, Xie GH. Alkali-based pretreatments distinctively extract lignin and pectin for enhancing biomass saccharification by altering cellulose features in sugar-rich Jerusalem artichoke stem. Bioresour Technol. 2016;208:31-41.

12. Song Y, Wi SG, Kim HM, Bae H. Cellulosic bioethanol production from Jerusalem artichoke (Helianthus tuberosus L.) using hydrogen peroxideacetic acid (HPAC) pretreatment. Bioresour Technol. 2016;214:30-6.

13. Gunnarsson IB, Svensson SE, Johansson E, Karakashev D, Angelidaki I. Potential of Jerusalem artichoke (Helianthus tuberosus L.) as a biorefinery crop. Ind Crop Prod. 2014;56:231-40.

14. Li C, Aston JE, Lacey JA, Thompson VS, Thompson DN. Impact of feedstock quality and variation on biochemical and thermochemical conversion. Renew Sustain Energy Rev. 2016;65:525-36.

15. Himmel ME, Ding S, Johnson DK, Adney WS, Nimlos MR, Brady JW, Foust TD. Biomass recalcitrance: engineering plants and enzymes for biofuels production. Science. 2007;315:804-7.

16. Deng J. Control problems of grey systems. Syst Control Lett. 1982;1:288-94.

17. Payne CE, Wolfrum EJ. Rapid analysis of composition and reactivity in cellulosic biomass feedstocks with near-infrared spectroscopy. Biotechnol Biofuels. 2015;8:43.

18. Bruun S, Jensen JW, Magid J, Lindedam J, Engelsen SB. Prediction of the degradability and ash content of wheat straw from different cultivars using near infrared spectroscopy. Ind Crop Prod. 2010;31:321-6.

19. Wu L, Li M, Huang J, Zhang H, Zou W, Hu S, Li Y, Fan C, Zhang R, Jing H, et al. A near infrared spectroscopic assay for stalk soluble sugars, bagasse enzymatic saccharification and wall polymers in sweet sorghum. Bioresour Technol. 2015;177:118-24.

20. Huang J, Xia T, Li A, Yu B, Li Q, Tu Y, Zhang W, Yi Z, Peng L. A rapid and consistent near infrared spectroscopic assay for biomass enzymatic digestibility upon various physical and chemical pretreatments in Miscanthus. Bioresour Technol. 2012:121:274-81.

21. Wei M, Geladi P, Lestander TA, Xie G, Xiong S. Multivariate modelling on biomass properties of cassava stems based on an experimental design. Anal Bioanal Chem. 2015;407:5443-52.

22. Yang Z, Li K, Zhang M, Xin D, Zhang J. Rapid determination of chemical composition and classification of bamboo fractions using visible-near infrared spectroscopy coupled with multivariate data analysis. Biotechnol Biofuels. 2016:9:35
23. Li M, Wang J, Du F, Diallo B, Xie GH. High-throughput analysis of chemical components and theoretical ethanol yield of dedicated bioenergy sorghum using dual-optimized partial least squares calibration models. Biotechnol Biofuels. 2017;10:206.

24. Xu F, Yu J, Tesso T, Dowell F, Wang D. Qualitative and quantitative analysis of lignocellulosic biomass using infrared techniques: a mini-review. Appl Energy. 2013;104:801-9.

25. Chadwick DT, McDonnell KP, Brennan LP, Fagan CC, Everard CD. Evaluation of infrared techniques for the assessment of biomass and biofuel quality parameters and conversion technology processes: a review. Renew Sustain Energy Rev. 2014;30:672-81.

26. Yun YH, Liang YZ, Xie GX, Li HD, Cao DS. A perspective demonstration on the importance of variable selection in inverse calibration for complex analytical systems. Analyst. 2013;138:6412-21.

27. Li H, Liang Y, Xu Q, Cao D. Model population analysis for variable selection. J Chemometr. 2010;24:418-23.

28. Li H, Liang Y, Cao D, Xu Q. Model-population analysis and its applications in chemical and biological modeling. TrAC Trends Anal Chem. 2012;38:154-62.

29. Li H, Liang Y, Xu Q, Cao D. Key wavelengths screening using competitive adaptive reweighted sampling method for multivariate calibration. Anal Chim Acta. 2009:648:77-84

30. Li H, Xu Q, Liang Y. Random frog: an efficient reversible jump Markov Chain Monte Carlo-like approach for variable selection with applications to gene selection and disease classification. Anal Chim Acta. 2012;740:20-6.

31. Wang Y, Fan C, Hu H, Li Y, Sun D, Wang Y, Peng L. Genetic modification of plant cell walls to enhance biomass yield and biofuel production in bioenergy crops. Biotechnol Adv. 2016;34:997-1017.

32. Wolfrum EJ, Ness RM, Nagle NJ, Peterson DJ, Scarlata CJ. A laboratoryscale pretreatment and hydrolysis assay for determination of reactivity in cellulosic biomass feedstocks. Biotechnol Biofuels. 2013;6:162.

33. Xiao L, Wei H, Himmel ME, Jameel H, Kelley SS. NIR and Py-mbms coupled with multivariate data analysis as a high-throughput biomass characterization technique: a review. Front Plant Sci. 2014;5:388.

34. Guimarães CC, Simeone MLF, Parrella RAC, Sena MM. Use of NIRS to predict composition and bioethanol yield from cell wall structural components of sweet sorghum biomass. Microchem J. 2014;117:194-201.

35. Schwanninger M, Rodrigues J, Fackler K. A review of band assignments in near infrared spectra of wood and wood components. J Near Infrared Spectrosc. 2011;19:287.

36. Fagan CC, Everard CD, McDonnell K. Prediction of moisture, calorific value, ash and carbon content of two dedicated bioenergy crops using near-infrared spectroscopy. Bioresour Technol. 2011;102:5200-6.

37. Martin AP, Palmer WM, Byrt CS, Furbank RT, Grof CP. A holistic highthroughput screening framework for biofuel feedstock assessment that characterises variations in soluble sugars and cell wall composition in Sorghum bicolor. Biotechnol Biofuels. 2013;6:186.

38. Zhao H, Li Q, He J, Yu J, Yang J, Liu C, Peng J. Genotypic variation of cell wall composition and its conversion efficiency in Miscanthus sinensis, a potential biomass feedstock crop in China. GCB Bioenergy. 2014;6:768-76.

39. Williams CL, Westover TL, Emerson RM, Tumuluru JS, Li C. Sources of biomass feedstock variability and the potential impact on biofuels production. Bioenergy Res. 2016:9:1-14.

40. Bhandari HS, Walker DW, Bouton JH, Saha MC. Effects of ecotypes and morphotypes in feedstock composition of switchgrass (Panicum virgatum L.). GCB Bioenergy. 2014;6:26-34.

41. Feltus FA, Vandenbrink JP. Bioenergy grass feedstock: current options and prospects for trait improvement using emerging genetic, genomic, and systems biology toolkits. Biotechnol Biofuel. 2012;5:80.

42. Huang JF, Li Y, Wang YT, Chen YY, Liu MY, Wang YM, Zhang R, Zhou SG, Li $J Y, T u$ YY, Hao B, Peng LC, Xia T. A precise and consistent assay for major wall polymer features that distinctively determine biomass saccharification in transgenic rice by near-infrared spectroscopy. Biotechnol Biofuels. 2017;10:294.

43. Hames B, Ruiz R, Scarlata C, Sluiter J, Templeton D. Preparation of samples for compositional analysis. NREL/TP-510-42620. 2008 
44. Sluiter A, Hames B, Ruiz R, Scarlata C, Sluiter J, Templeton D. Determination of Ash in biomass. NREL/TP-510-42622. 2008.

45. Sluiter A, Hames B, Hyman D, Payne C, Ruiz R, Scarlata C, Sluiter J, Templeton D, Wolfe J. Determination of total solids in biomass and total dissolved solids in liquid process samples. NREL/TP-510-42621. 2008.
46. Sluiter A, Ruiz R, Scarlata C, Sluiter J, Templeton D Determination of extractives in biomass. NREL/TP-510-42619. 2008.

47. Sluiter A, Hames B, Ruiz R, C. Scarlata, Sluiter J, Templeton D, Crocker D. Determination of structural carbohydrates and lignin in biomass. NREL/ TP-510-42618. 2012.
Ready to submit your research? Choose BMC and benefit from:

- fast, convenient online submission

- thorough peer review by experienced researchers in your field

- rapid publication on acceptance

- support for research data, including large and complex data types

- gold Open Access which fosters wider collaboration and increased citations

- maximum visibility for your research: over $100 \mathrm{M}$ website views per year

At BMC, research is always in progress.

Learn more biomedcentral.com/submissions 